Check for updates

Cite this: RSC Adv., 2017, 7, 26145

\title{
Superhydrophobic surfaces on brass substrates fabricated via micro-etching and a growth process
}

Received 21st March 2017

Accepted 9th May 2017

DOI: $10.1039 / \mathrm{c} 7 \mathrm{ra03308g}$

rsc.li/rsc-advances

\author{
Junyang Tan, Junjie Hao, Zhenqiang An and Changsheng Liu (D)*
}

Constructing artificial superhydrophobic surfaces is an effective strategy for improving the properties of traditional materials. In this paper, we report an easy method for preparing superhydrophobic surfaces on brass to expand its field of application. The micro-nanostructure on the brass surface was constructed via micro-etching technology, and the resulting surface energy was reduced via stearic acid modification. The surface was analyzed using scanning electron microscopy and X-ray photoelectron spectroscopy. The brass surface was converted from hydrophilic to superhydrophilic to superhydrophobic. Bending and abrasion tests were used to characterize the mechanical durability of the surface. Additionally, the obtained surface exhibited a superior self-cleaning performance. We propose that this method has a promising future for superhydrophobic surface fabrication and can expand the applications of brass.

\section{Introduction}

The surfaces of many plants and insects, such as rose petals, rice leaves, and butterfly wings, have attracted much interest because of their excellent water-repellent traits. ${ }^{1-4}$ Such surfaces have contact angles (CA) $>150^{\circ}$ and sliding angles (SA) $<10^{\circ}$ and are called superhydrophobic surfaces. ${ }^{5,6}$ The lotus leaf is the most wellknown superhydrophobic surface and has been studied intensively. Neinhuis and Barthlott used scanning electron microscopy to investigate the lotus leaf and found that it possesses a dual hierarchical structure and the surface is covered with low surface energy waxy organics; ${ }^{7}$ they proposed that both the special surface morphology and the chemical composition are essential for superhydrophobicity. Many methods for fabricating artificial superhydrophobic surfaces have been reported, including selfassembly, ${ }^{8-10}$ a sol-gel technique, ${ }^{11-13}$ electrochemical reaction and deposition, ${ }^{14-16}$ and laser fabrication. ${ }^{17-19}$ Because of their unique wetting behaviors, superhydrophobic surfaces have enormous potential for applications in a wide variety of fields. For example, using constructed superhydrophobic surfaces, Chen et al. separated oil/water mixtures efficiently, ${ }^{20}$ and Xue et al. removed microbubbles from aqueous media. ${ }^{21}$

Brass is a common copper alloy ( $\mathrm{Cu}-\mathrm{Zn})$ and is widely used in daily life and industrial production because of its particular physical and mechanical properties, including its high thermal conductivity, low friction, good anti-corrosion ability, and good casting properties. ${ }^{22}$ As industry and technology develop, brass is expected to have even more applications. It is thus necessary to improve the properties of brass, such as its resistance to corrosion

Key Laboratory for Anisotropy and Texture of Materials, Ministry of Education, Northeastern University, Shenyang 110819, China. E-mail: neujohnson@163.com; csliu@mail.neu.edu.cn in seawater and its self-cleaning ability in dusty conditions so that it can be used in various harsh environments. Constructing superhydrophobic coatings on brass is an effective method for improving its properties and expanding its applications. However, few methods for preparing a superhydrophobic brass surface have been reported. Jie et al. used a combined approach for preparing a superhydrophobic surface via etching with an aqueous solution of $\mathrm{FeCl}_{3}$ for $45 \mathrm{~min}$, heating at $350{ }^{\circ} \mathrm{C}$ for $25 \mathrm{~min}$, and then modifying the surface with stearic acid. ${ }^{23}$ Ta et al. obtained a nanosecond laser textured superhydrophobic metallic surface using a fiber laser with a nominal beam spot of about $21 \mu \mathrm{m}$ at a scanning speed of $75 \mathrm{~mm} \mathrm{~s}^{-1} .{ }^{24}$ However, the low efficiency of these reported methods has restricted the widespread application of superhydrophobic brass surfaces. It is a practical and valuable task to improve the preparation efficiency.

In this study, a superhydrophobic surface was fabricated on brass using a simple micro-etching technique. Numerous rough structures on the micro/nanometer scale were achieved, and the free energy of the surface was reduced using stearic acid modification. The as-prepared surface had great mechanical durability and a self-cleaning ability. There was only slight damage on the substrate compared to other traditional etching methods used for fabricating superhydrophobic surfaces. Additionally, compared to other techniques for fabricating superhydrophobic brass surface, this preparation method is highly effective and easily controlled. We believe that the present method may expand the prospective applications of brass.

\section{Materials and methods}

\subsection{Materials}

Brass plates with a thickness of $0.5 \mathrm{~mm}$ were cut into $100 \mathrm{~mm} \times$ $60 \mathrm{~mm}$ samples. Hydrochloric acid ( $\mathrm{HCl})$, sulfuric acid $\left(\mathrm{H}_{2} \mathrm{SO}_{4}\right)$, 
sodium chloride $(\mathrm{NaCl})$, and sodium sulphate $\left(\mathrm{Na}_{2} \mathrm{SO}_{4}\right)$ were supplied by Tianjin Kemiou Chemical Reagent Co., Ltd. Stearic acid (STA) was purchased from Tianjin Fuyu Fine Chemical Co., Ltd. All other chemicals were analytical grade and were used as received.

\subsection{Sample preparation}

To remove organic contaminants from the surface, the brass plate was first polished with sandpaper (from 400 to 1200 grit) and ultrasonically washed with acetone, ethanol, and deionized water for $6 \mathrm{~min}$ each. The sample was then immersed in a solution of $10 \mathrm{wt} \% \mathrm{H}_{2} \mathrm{SO}_{4}$ for $30 \mathrm{~s}$ to remove oxide films, cleaned with deionized water and ethanol, and then air-dried. A reaction immersion solution containing $1 \mathrm{M} \mathrm{HCl}$ was then prepared for brass micro-etching, and $\mathrm{NaCl}$ and $\mathrm{Na}_{2} \mathrm{SO}_{4}$ were added in a mass ratio of $6: 1$. The cleaned brass sample was dipped in the immersion solution at room temperature for $10 \mathrm{~s}$. Without drying, the dipped sample was removed from solution and heated in a thermostatic drying oven at a temperature of $110{ }^{\circ} \mathrm{C}$ for the micro-etching process to occur. The reaction time was varied from 1 to $10 \mathrm{~min}$ to obtain an optimum surface texture required for superhydrophobicity. After the reaction, the sample was removed from the oven, cooled at room temperature for $30 \mathrm{~s}$, and then dipped in a $0.1 \mathrm{M}$ STA ethanol solution for 2 minutes to modify the surface. Ultimately, a superhydrophobic brass surface was obtained and dried with cold air.

\subsection{Characterization}

The CAs for water were measured using the sessile drop method with a $3 \mu \mathrm{L}$ droplet admeasuring apparatus (JCY-2, Fangrui) under ambient conditions. All measurements of each sample were repeated at a minimum of five different positions and then averaged. The morphological micro-nanostructure of the as-prepared surface was observed using scanning electron microscopy (SEM, FEI NanoSEM 430). The sample surface roughness was characterized using a laser confocal scanning microscope (LSCM, Olympus OLS3100). The chemical composition of the surface was analyzed using X-ray photoelectron spectroscopy (XPS, K-Alpha) and X-ray diffraction (XRD, Bruke D8 Advance).

\section{Results and discussion}

\subsection{Surface morphology and chemical composition}

The formation process of the superhydrophobic brass surface is given in Fig. 1. First, the immersion solution reacted with the brass substrate, and numerous tiny cavities formed on the surface. During reaction, zinc was the only element etched while copper remained as an elementary substance; thus, the process is called micro-etching. At high temperature, $\mathrm{NaCl}$ crystallized on the substrate and became embedded in the cavities. Each of these two processes contributed to the rough structure. Finally, the STA modification endowed the as-etched surface with excellent superhydrophobicity because the existence of $-\mathrm{CH}_{3}$ and $-\mathrm{CH}_{2}$ - groups significantly decreased the free energy of the surface.

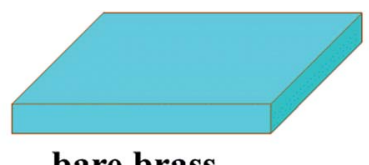

bare brass

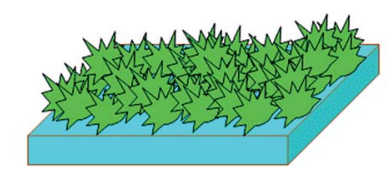

micro-etched brass
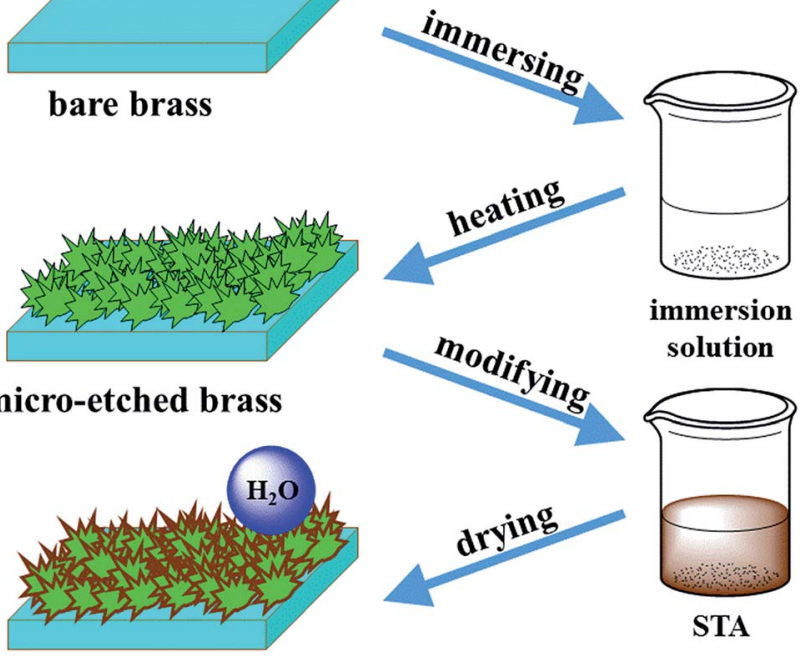

immersion solution

modified brass

Fig. 1 Schematic illustration of the process for fabricating a superhydrophobic surface on the brass substrate.

The wettability and morphology of the superhydrophobic surface were affected by several factors, including temperature, $\mathrm{pH}$, immersion solution concentration, and etching time. ${ }^{25-29}$ Herein, the effect of the micro-etching time was the primary consideration. The effect of the micro-etching time on the wettability of the brass substrate was studied using CA measurements. Fig. 2 illustrates the CA variation of the modified surface for different lengths of micro-etching time. The surface that was micro-etched for $1 \mathrm{~min}$ was hydrophobic with a CA of $135.7^{\circ}$. The CA increased with an increase in microetching time. When the sample was micro-etched for $6 \mathrm{~min}$, the CA improved to a maximum of $152.4^{\circ}$, and the SA was $5.3^{\circ}$, indicating that the modified sample had excellent superhydrophobicity. Following the process of micro-etching, the CAs

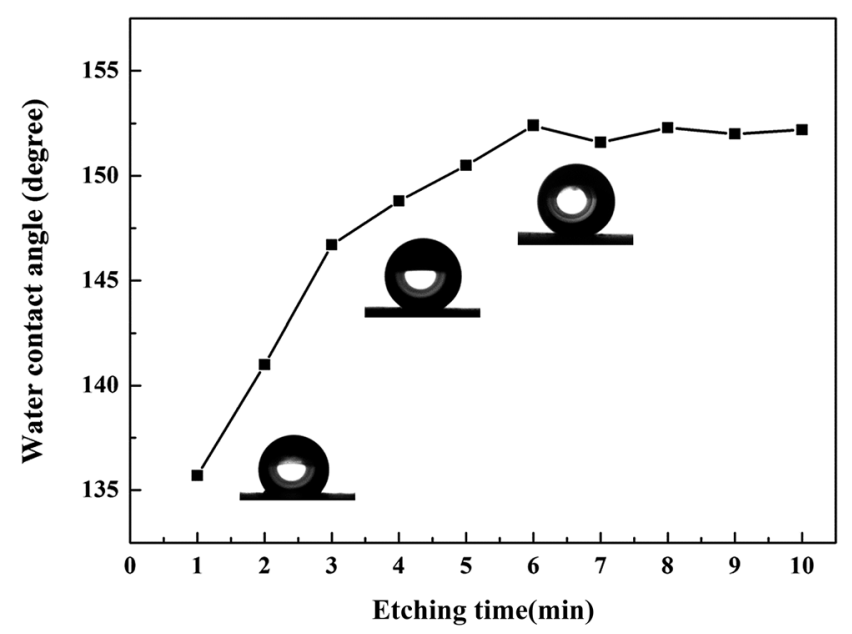

Fig. 2 Effects of the micro-etching time on the CAs of the asprepared surfaces. 
remained over $150^{\circ}$, and the range of change was relatively small. Therefore, it was concluded that the optimal etching time was $6 \mathrm{~min}$ in terms of efficiency and cost.

It is known that surface morphology affects the wettability of the surface. Fig. 3 shows the SEM images of the STA-modified surface with different lengths of micro-etching time at different magnifications. When the etching time was as short as $1 \mathrm{~min}$, a rough morphology was observed on the substrate (Fig. 3a and b). Since the brass composition was not homogeneous, there were unequal etching rates at different positions of the plate. ${ }^{30}$ Thus the micrometer-scale structures were unevenly distributed on the surface and revealed good roughness. When the reaction time was increased to $4 \mathrm{~min}$, the sample had a large and uniformly distributed amount of grass-like micro sheets (Fig. 3c and d), resulting in a roughness hierarchy. With this reaction process, such micro sheets grew upwards gradually and became interconnected, forming clusters. As shown in Fig. 3e, after $6 \mathrm{~min}$ of reaction time, the agglomeration process continued, and numerous hemispherical rough structures were constructed on the sample. With higher magnification (Fig. 3f), dandelion-like clusters with sharp edges were seen. These clusters had an average size of $10 \mu \mathrm{m}$, and air was trapped easily in the spaces between the clusters.

The surface roughness $\left(R_{\mathrm{a}}\right)$ further revealed changes in the morphology. Fig. 4 shows the LSCM images, the $R_{\mathrm{a}}$ profile curves, and the water drop photographs of the surface of each the bare, micro-etched, and superhydrophobic brass. The bare brass sample had an apparently smooth topography with a $R_{\mathrm{a}}$ value of $0.397 \mu \mathrm{m}$, and on the surface of the bare brass, the water droplet had a CA of $61.3^{\circ}$. After micro-etching, many coarse structures formed on the samples and dramatically increased the $R_{\mathrm{a}}$ to $2.635 \mu \mathrm{m}$. The water droplets spread on the as-etched surface with a CA of less than $5^{\circ}$. After modification with STA, the surface was converted from superhydrophilic to superhydrophobic. The water droplets formed spherical shapes and had a CA of $152.4^{\circ}$. Moreover, because of the formation of the richer topographic characteristics caused by STA, the $R_{\mathrm{a}}$ further increased to $3.307 \mu \mathrm{m}$, and this indicates that air was trapped easily in the interspacings of the rough surface.

To further understand the superhydrophobicity, the wetting behaviors of the superhydrophobic brass surface were described using the Cassie-Baxter model, ${ }^{31}$ where large amounts of air were trapped in the micro-nanostructure. The CA is mathematically denoted as:

$$
\cos \theta_{\mathrm{r}}=f \cos \theta+f-1
$$

where $f$ represents the contact area fraction of the solid/liquid interface. $\theta_{\mathrm{r}}\left(152.4^{\circ}\right)$ and $\theta\left(107^{\circ}\right)^{32}$ represent the CAs on the superhydrophobic brass surface and the primary surface, respectively. For our sample, $f$ was calculated as 0.161 , demonstrating that up to $83.9 \%$ of the contact-area between the
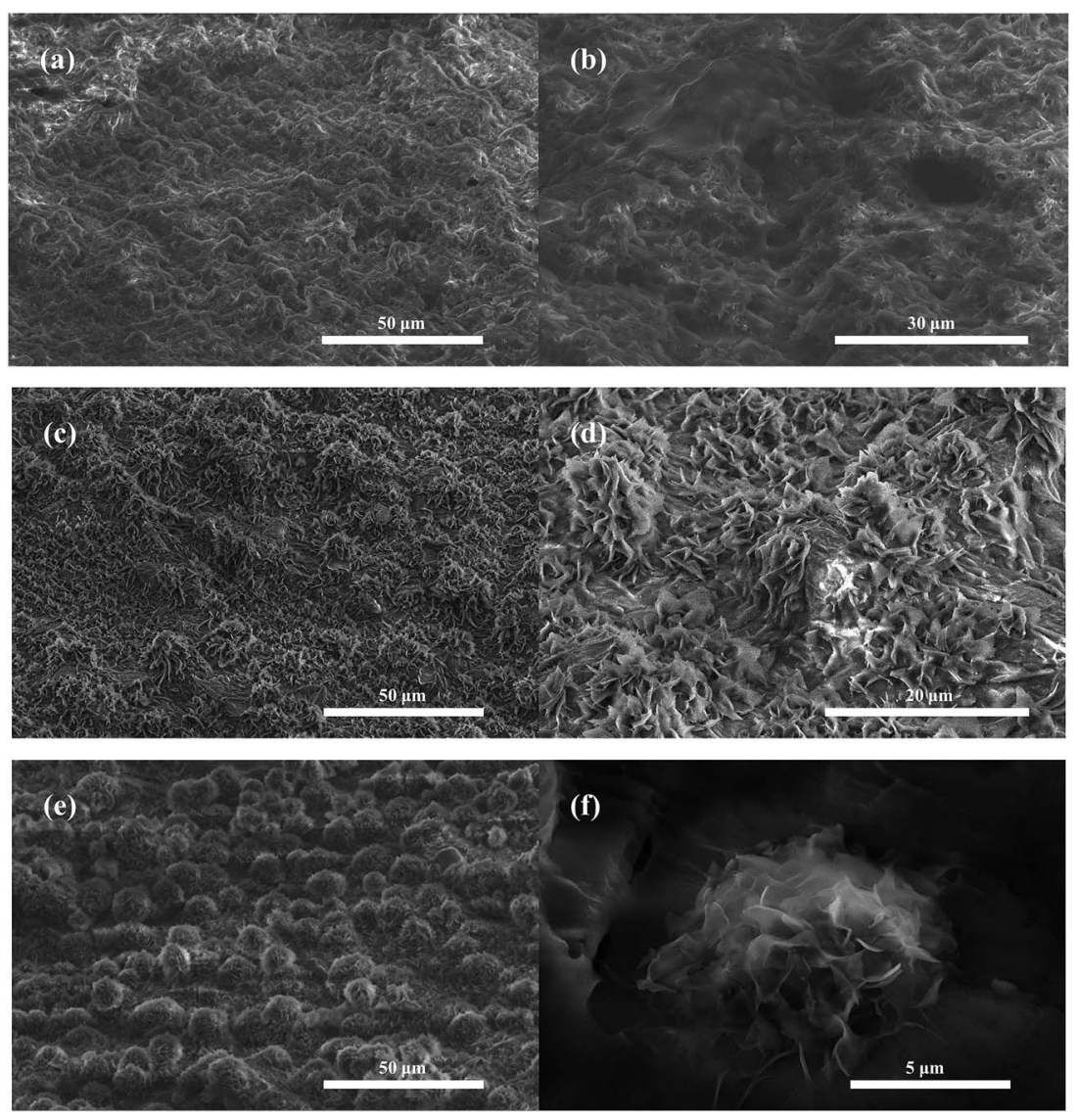

Fig. 3 SEM images of the as-prepared surfaces at different micro-etching times of (a and b) 1 min, (c and d) 3 min, and (e and f) 6 min. 


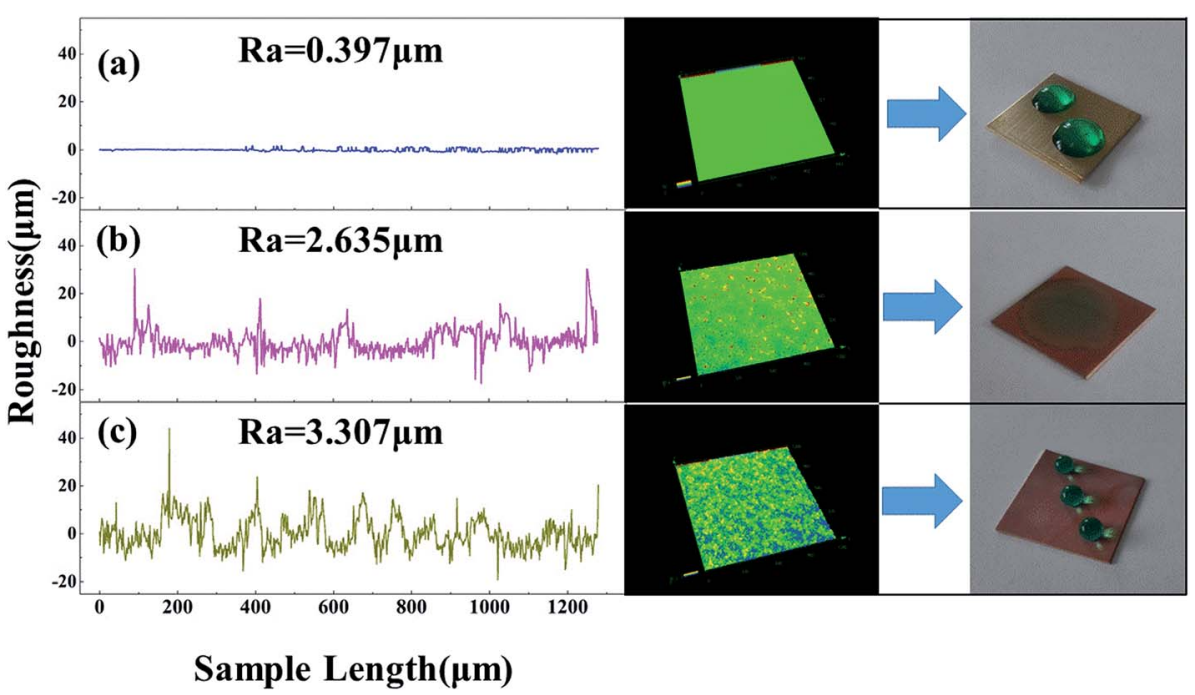

Fig. 4 LSCM topographical images, surface roughness profile curves, and optical photographs of the water drops on the surface of each the bare (a), micro-etched (b), and superhydrophobic (c) brass.

water droplet and the samples was composed of air. It is generally known that air is perfectly superhydrophobic, so entrapped air largely enhance the capacity of the surface to repel water.

XRD and XPS measurements were used to analyze the chemical composition of the as-prepared surfaces. Fig. 5a compares the XRD patterns of the bare brass surface (A) and the micro-etched brass surface (B) in a $2 \theta$ scan range of $30-90^{\circ}$. The four diffraction peaks at $2 \theta=42.32^{\circ}, 49.27^{\circ}, 72.24^{\circ}$, and $87.45^{\circ}$ correspond to $\mathrm{Cu}_{0.64} \mathrm{Zn}_{0.36}$ (JCPDS Card No. 50-1333), which came from the brass substrate. After micro-etching, however, new reflections appeared at $2 \theta=31.69^{\circ}, 45.45^{\circ}$, and $75.30^{\circ}$ corresponding to $\mathrm{NaCl}$ (JCPDS Card No. 05-0628), which indicate that $\mathrm{NaCl}$ crystallized on the brass surface during the reaction.

XPS spectroscopy was used to characterize the changes of the surface chemical composition after the STA modification. The full spectrum of the modified brass surface is shown in Fig. 5b, and the presence of $\mathrm{C}, \mathrm{O}, \mathrm{Na}, \mathrm{Cl}$, and $\mathrm{Zn}$ were clearly observed. The surface also had a weak signal for $\mathrm{Cu} 2 \mathrm{~s}$ (Fig. 5c). In Fig. 5d, the $\mathrm{O}$ 1s spectrum consisted of two peaks: at $535.5 \mathrm{eV}$ and $530.8 \mathrm{eV}$. The peak at $535.5 \mathrm{eV}$ is usually assigned to chemisorbed or adsorbed oxygen species such as $\mathrm{O}_{2}, \mathrm{H}_{2} \mathrm{O}$, and $\mathrm{ZnO}(\mathrm{OH})$ because of surface hydroxyl groups. ${ }^{33}$ The peak located at $530.8 \mathrm{eV}$ is due to the $\mathrm{O}^{2-}$ in $\mathrm{Zn}-\mathrm{O}$, and this demonstrates that the STA molecules reacted with the $-\mathrm{OH}$ groups of the $\mathrm{Zn}$. The C 1s high-resolution spectrum is shown in Fig. 5e. The peak at $283.9 \mathrm{eV}$ was attributed to carbon atoms in $\mathrm{C}-\mathrm{C}$ or $\mathrm{C}-\mathrm{H}$, and the peak at $287.6 \mathrm{eV}$ was assigned to the carbon atom in $\mathrm{O}-\mathrm{C}=$ $\mathrm{O}$, which confirms the existence of a long-chain aliphatic group containing-COO-. The combined analyses of the $\mathrm{O} 1 \mathrm{~s}$ and $\mathrm{C} 1 \mathrm{~s}$ spectra leads us to hypothesize that the STA molecules have a good affinity for the etched brass substrate via the condensation reaction, in which the carboxyl group $(\mathrm{COO}-\mathrm{H})$ combines with the hydroxyl group $(-\mathrm{OH})$ in the brass and releases water, forming $\mathrm{CH}_{3}\left(\mathrm{CH}_{2}\right)_{16} \mathrm{COO}$-brass. ${ }^{34,35}$

\subsection{Mechanical and thermal stability}

Mechanical durability plays a key role in the use of superhydrophobic surfaces in industrial applications..$^{36-38}$ In this paper, we used a bending test and an abrasion test to assess the stability of the superhydrophobicity. Fig. 6a and b shows images from two views of water droplets on the bent superhydrophobic brass. It can be clearly seen that after bending to an angle of $60^{\circ}$, the bent surface still had excellent superhydrophobic properties. The droplets in the bent area retained a spherical shape and slid off freely with little resistance. The SEM image of the fold is shown in Fig. 6c. It can be seen that only a small portion of the micro-nanostructure was lost from the substrate while most of the original structure was still in place, and what remained had no observable changes. Thus, the as-prepared sample maintained its water repellency.

The steps of the abrasion test are shown in Fig. 7a. The asprepared sample was pushed on 1200 grit SiC sandpaper at a speed of $2 \mathrm{~cm} \mathrm{~s}^{-1}$ with a weight of $50 \mathrm{~g}$ which provided $2.5 \mathrm{kPa}$ of applied pressure. The distance moved was $40 \mathrm{~cm}$ in one direction. After abrasion, the CA decreased to $137.7^{\circ}$. Interestingly, the droplet stuck to the surface and did not roll off even when the sample was positioned vertically, and this is called sticky hydrophobicity. ${ }^{39} \mathrm{Fig} .7 \mathrm{~b}$ and c shows the SEM images of the superhydrophobic brass surface after abrasion. The upper part of the hemispherical structure was scraped, causing partial damage to the morphology. Moreover, as the upper layer of STA molecules were bound to each other only via van der Waals interactions, partially adsorbed STA molecules rubbed off the sample under mechanical stress and resulted in the change in the surface free energy. ${ }^{5}$ Thus, the abrasion process affected the wetting behavior of the brass surface and was responsible for the switch from non-sticky superhydrophobicity to sticky hydrophobicity.

In many applications, brass surfaces are exposed to heat, and so it is necessary to assess the thermal stability of the 

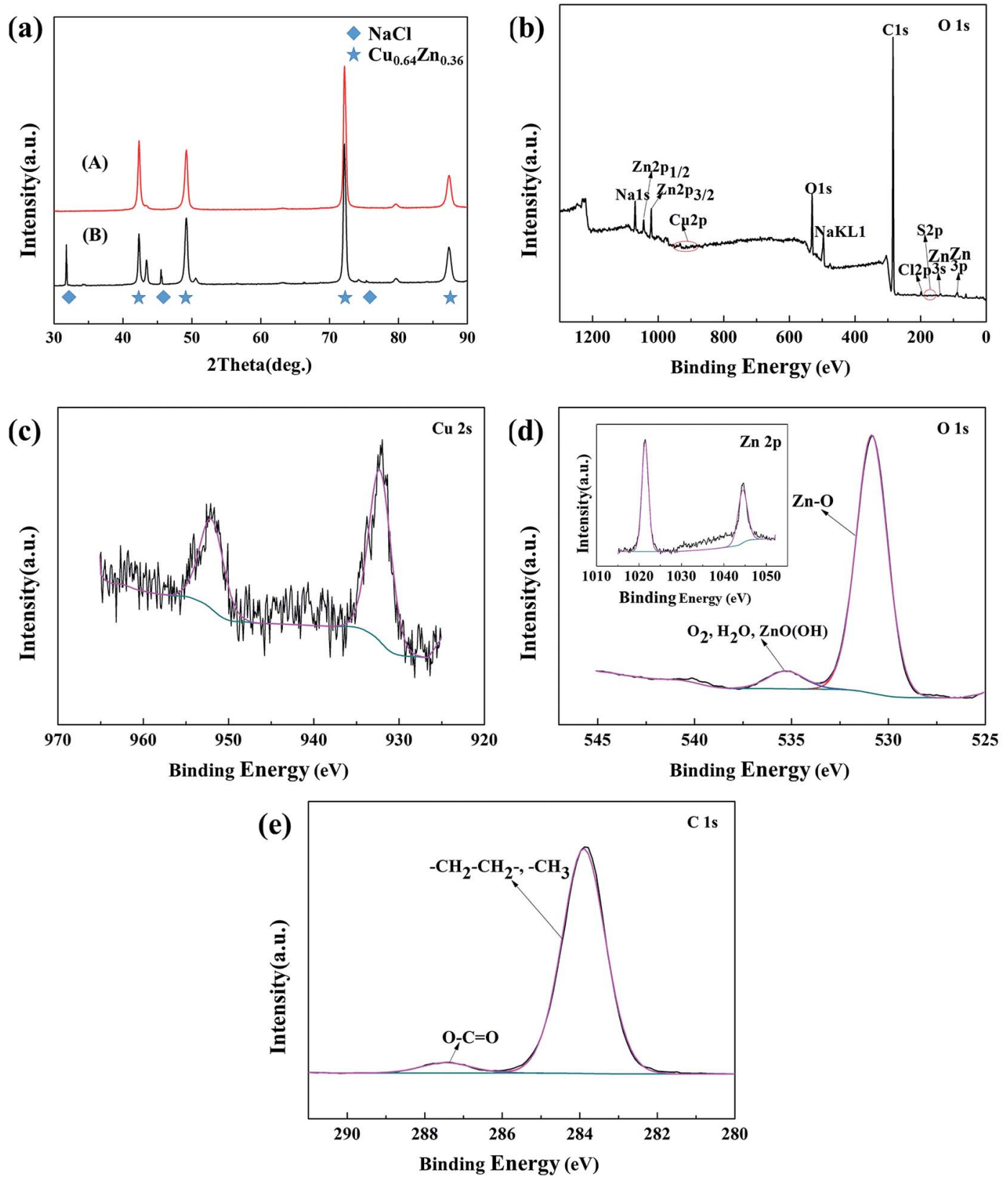

Fig. 5 XRD patterns of the brass substrate before and after micro-etching (a). XPS spectra of the superhydrophobic brass surface including (b) the survey spectrum, (c) the Cu 2 s region, (d) the $O 1$ s region with an inset of $\mathrm{Zn} 2 \mathrm{p}$, and (e) the $\mathrm{C}$ 1s region.

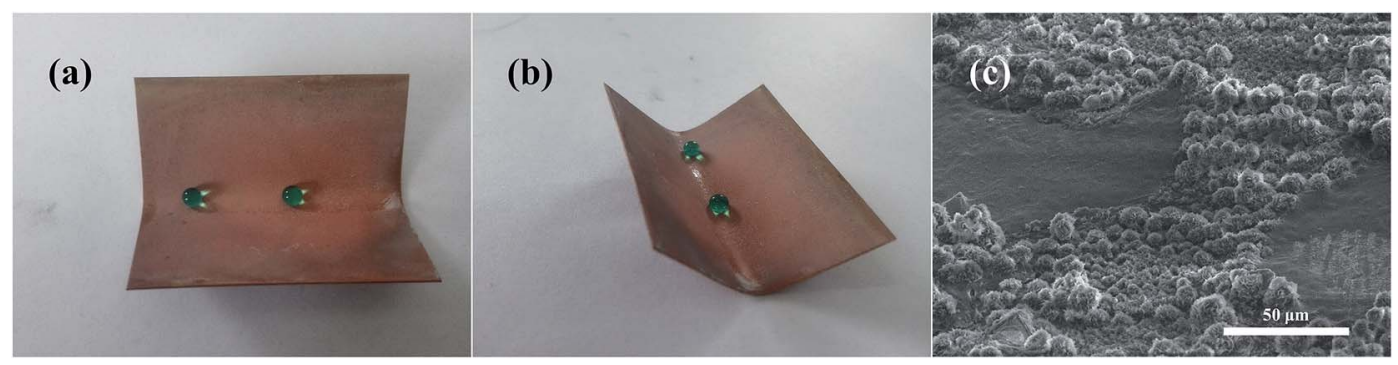

Fig. 6 (a and b) Optical photographs showing the shape of the water droplets on the bent surface. (c) SEM image of the fold. 

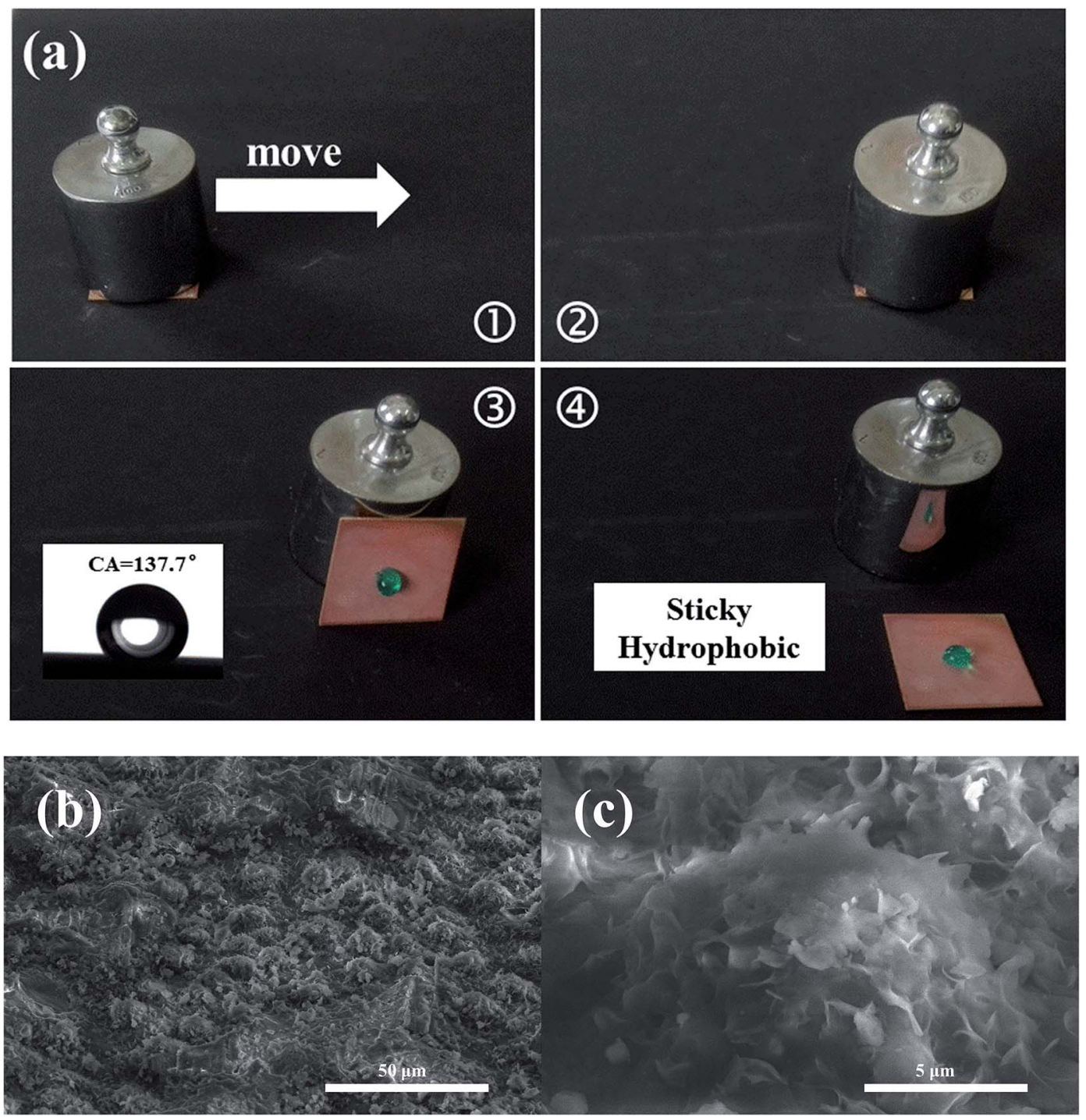

Fig. 7 (a) Steps of the abrasion test on the superhydrophobic brass surface. (b and c) SEM images of the superhydrophobic brass surface after abrasion.

superhydrophobic brass surface at high temperatures. To investigate this, the as-prepared samples were annealed from 50 to $225{ }^{\circ} \mathrm{C}$ for $5 \mathrm{~min}$, and the CAs are given in Fig. 8. When the temperature was below $100{ }^{\circ} \mathrm{C}$, the values of the CA were above $150^{\circ}$, indicating that the surface still retained a sufficient amount of its superhydrophobicity. Then the CA decreased significantly with an increase in temperature, and this was due to the STA decomposition/oxidation. Thus, the surface we fabricated may not be applied in strong thermal environments. This is a problem we want to solve in future work. Additionally, after being exposed to air for one month, the as-prepared surface still retained its superhydrophobicity, and the CA had no remarkable change.

\subsection{Self-cleaning property}

Self-cleaning is defined as the spontaneous roll-to-clean strategy of a surface, ${ }^{40}$ and this is illustrated in Fig. 9a. To test the self-

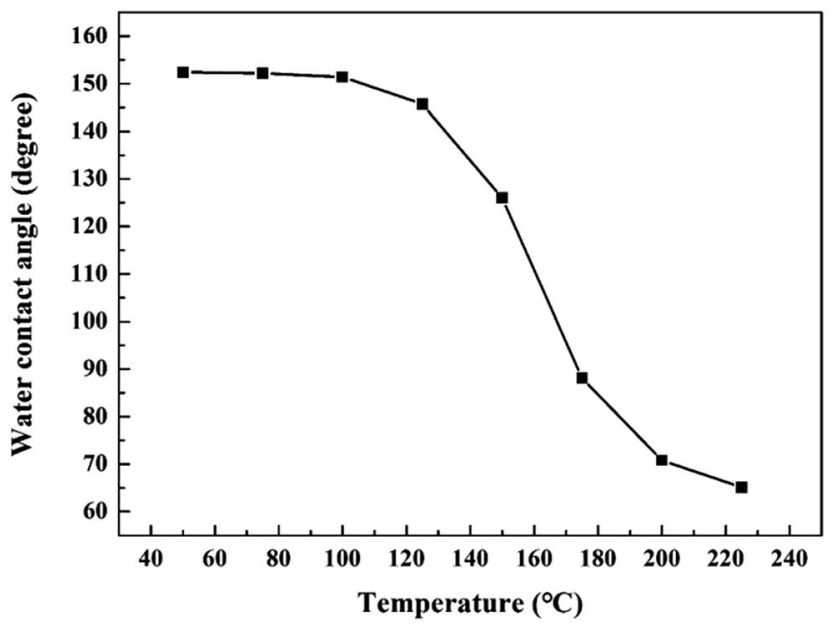

Fig. 8 Thermal stability test results for the superhydrophobic brass surface. 


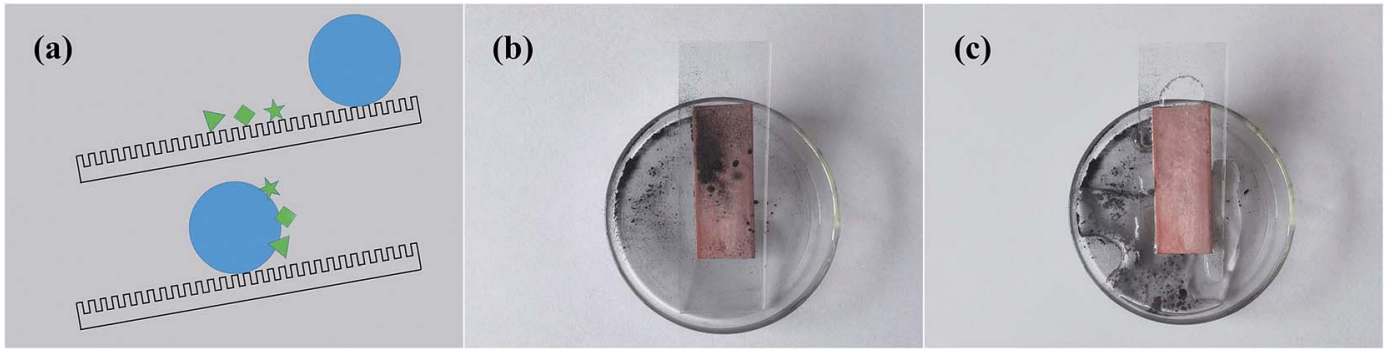

Fig. 9 (a) Model of self-cleaning, and ( $b$ and $c$ ) the self-cleaning behavior of the superhydrophobic brass surface.

cleaning property of the as-prepared surface, we covered it with carbon dust to simulate an extremely dusty environment (Fig. 9b). As the water droplets rolled off the surface quickly, the dust particles were carried away, and the sample was clean within a short time (Fig. 9c). This can be explained by the combined effects of the high hydrophobicity of the surface and the poor adhesive force between the carbon dust and the asprepared sample. Thus, the self-cleaning ability of the asprepared surface could readily shelter substrates from pollution in actual applications.

\section{Conclusions}

In this study, a superhydrophobic brass surface with a CA of $152.4^{\circ}$ was successfully fabricated using a simple and fast micro-etching technique followed by STA modification. The micro-etching time had a major impact on the surface hydrophobicity, and the best effect was with a reaction time of $6 \mathrm{~min}$. The whole fabrication process was finished within $10 \mathrm{~min}$, and all of the required equipment and materials were accessible and affordable. After the bending test and the abrasion test, the asprepared surface retained good water-repellency and showed good mechanical resistance. Moreover, the surface transformed from non-sticky superhydrophobic to sticky hydrophobic after abrasion. In addition, carbon dust was easily removed from the surface under the action of rolling water drops, and this indicates an excellent self-cleaning ability. We propose that the present method is an effective approach for constructing superhydrophobic surfaces on brass substrates and can be further extended to a variety of industrial applications.

\section{Acknowledgements}

The authors are very grateful for the support from the National Training Program of Innovation and Entrepreneurship for Undergraduates (No. 201610145034) and the Joint Funds of NSFC-Liaoning (U1508213). The first author (Junyang Tan) and the second author (Junjie Hao) contributed equally to this work.

\section{References}

1 J. Long, P. Fan, D. Gong, D. Jiang, H. Zhang, L. Li and M. Zhong, ACS Appl. Mater. Interfaces, 2015, 7, 9858-9865.

2 C. Liu, J. Ju, Y. Zheng and L. Jiang, ACS Nano, 2014, 8, 13211329.
3 G. D. Bixler and B. Bhushan, Nanoscale, 2014, 6, 76-96.

4 J. K. Oh, X. Lu, Y. Min and M. Akbulut, ACS Appl. Mater. Interfaces, 2015, 7, 19274-19281.

5 F. Su and K. Yao, ACS Appl. Mater. Interfaces, 2014, 6, 87628770 .

6 S. Wooh, H. Huesmann, M. N. Tahir, M. Paven, K. Wichmann, D. Vollmer, W. Tremel, P. Papadopoulos and H. Butt, Adv. Mater., 2015, 27, 7338-7343.

7 W. Barthlott and C. Neinhuis, Planta, 1997, 202, 1-8.

8 J. V. I. Timonen, M. Latikka, L. Leibler and R. H. A. Ras, Science, 2013, 341, 253-257.

9 A. Checco, A. Rahman and C. T. Black, Adv. Mater., 2014, 26, 886-891.

10 Q. Li, Y. Wang, C. Rong, F. Zhang, Y. Liu, L. Chen, Q. Wang and C. Peng, Ceram. Int., 2016, 42, 2829-2835.

11 S. Cai, Y. Zhang, H. Zhang, H. Yan, H. Lv and B. Jiang, ACS Appl. Mater. Interfaces, 2014, 6, 11470-11475.

12 S. S. Latthe, C. Terashima, K. Nakata, M. Sakai and A. Fujishima, J. Mater. Chem. A, 2014, 2, 5548-5553.

13 M. Boudot, V. Gaud, M. Louarn, M. Selmane and D. Grosso, Chem. Mater., 2014, 26, 1822-1833.

14 S. Zhang, J. Huang, Y. Tang, S. Li, M. Ge, Z. Chen, K. Zhang and Y. Lai, Small, 2016, 13, 201600687.

15 T. Darmanin, E. T. Givenchy, S. Amigoni and F. Guittard, Adv. Mater., 2013, 25, 1378-1394.

16 Y. Wu, K. Liu, B. Su and L. Jiang, Adv. Mater., 2014, 26, 11241128.

17 L. B. Boinovich, A. G. Domantovskiy, A. M. Emelyanenko, A. S. Pashinin, A. A. Ionin, S. I. Kudryashov and P. N. Saltuganov, ACS Appl. Mater. Interfaces, 2014, 6, 2080-2085.

18 H. B. Jiang, Y. L. Zhang, D. D. Han, H. Xia, J. Feng, Q. D. Chen, Z. R. Hong and H. B. Sun, Adv. Funct. Mater., 2014, 24, 4595-4602.

19 Y. H. Yeong, C. Wang, K. J. Wynne and M. C. Gupta, ACS Appl. Mater. Interfaces, 2016, 8, 32050-32059.

20 X. Chen, Y. N. Liang, X. Z. Tang, W. Shena and X. Hu, Chem. Eng. J., 2017, 308, 18-26.

21 X. Xue, C. Yu, J. Wang and L. Jiang, ACS Nano, 2016, 10, 10887-10893.

22 C. Meran, Mater. Des., 2006, 27, 719-726.

23 H. Jie, Q. Xu, L. Wei and Y. Min, Corros. Sci., 2016, 102, 251-258.

24 D. V. Ta, A. Dunn, T. J. Wasley, R. W. Kay, J. Stringer, P. J. Smith, C. Connaughton and J. D. Shephard, Appl. Surf. Sci., 2015, 357, 248-254. 
25 H. Wang, J. Yu, Y. Wu, W. Shao and X. Xu, J. Mater. Chem. A, 2014, 2, 5010-5017.

26 L. Li, T. Huang, J. Lei, J. He, L. Qu, P. Huang, W. Zhou, N. Li and F. Pan, ACS Appl. Mater. Interfaces, 2015, 7, 1449-1457.

27 Q. Zhu, Y. Chu, Z. Wang, N. Chen, L. Lin, F. Liu and Q. Pan, J. Mater. Chem. A, 2013, 1, 5386-5393.

28 R. Gao, Q. Liu, J. Wang, X. Zhang, W. Yang, J. Liu and L. Liu, Chem. Eng. J., 2014, 241, 352-359.

29 W. Liu, Q. Xu, J. Han, X. Chen and Y. Min, Corros. Sci., 2016, 110, 105-113.

30 M. Qu, B. Zhang, S. Song, L. Chen, J. Zhang and X. Cao, Adv. Funct. Mater., 2007, 17, 593-596.

31 A. B. D. Cassie and S. Baxter, Trans. Faraday Soc., 1944, 40, 546-551.

32 Y. Qing, C. Yang, C. Hu, Y. Zheng and C. Liu, Appl. Surf. Sci., 2015, 326, 48-54.

33 Y. Qing, C. Yang, Z. Yu, Z. Zhang, Q. Hu and C. Liu, J. Electrochem. Soc., 2016, 163, D385-D391.
34 Y. Liu, X. Yin, J. Zhang, S. Yu, Z. Han and L. Ren, Electrochim. Acta, 2014, 125, 395-403.

35 Y. Huang, D. K. Sarkar and X. Chen, Appl. Surf. Sci., 2015, 327, 327-334.

36 S. S. Latthe, P. Sudhagar, A. Devadoss, A. M. Kumar, S. Liu, C. Terashima, K. Nakata and A. Fujishima, J. Mater. Chem. A, 2015, 3, 14263-14271.

37 X. Tian, T. Verho and R. H. A. Ras, Science, 2016, 352, 142143.

38 N. Wang, D. Xiong, Y. Deng, Y. Shi and K. Wang, ACS Appl. Mater. Interfaces, 2015, 7, 6260-6272.

39 Y. Lai, F. Pan, C. Xu, H. Fuchs and L. Chi, Adv. Mater., 2015, 262, 210-216.

40 S. S. Latthe, P. Sudhagar, C. Ravidhas, A. J. Christy, D. D. Kirubakaran, R. Venkatesh, A. Devadoss, C. Terashima, K. Nakata and A. Fujishima, CrystEngComm, 2015, 17, 2624-2628. 